\title{
Orthogonal frequency division multiplexing with amplitude shift keying subcarrier modulation as a reliable and efficient transmission scheme for self-mixing receivers
}

\author{
Jonas Kornprobst, Thomas J. Mittermaier, and Thomas F. Eibert \\ Chair of High-Frequency Engineering, Department of Electrical and Computer Engineering, Technical University of Munich, \\ 80290 Munich, Germany
}

Correspondence to: Jonas Kornprobst (j.kornprobst@tum.de)

Received: 21 December 2016 - Revised: 14 August 2017 - Accepted: 21 August 2017 - Published: 21 September 2017

\begin{abstract}
A new receiving scheme for self-mixing receivers is presented that overcomes the disadvantages of the selfheterodyne concept. Generally speaking, the self-mixing receiver offers immunity to phase noise and frequency offsets, especially at very high frequencies, since it does not require radio frequency local oscillators. Our proposed technique eliminates the drawbacks of the self-heterodyne transmission scheme, which are the poor power efficiency and the strong dependence on the continously transmitted carrier.

A nonlinear system of equations is constructed that describes a phase retrieval problem for the reconstruction of the original transmit signal before self-mixing. Two different solution strategies, with restrictions in time and frequency domain, are presented. As a consequence, the self-mixing equation system is shown to be solvable with some a-priori information about the transmit signal. With this novel approach, the transmitted information is distributed over the full available bandwidth, and there is no special dependence on a certain subcarrier for the down-conversion.

The general performance, regarding bit error ratio over signal to noise ratio, is improved by at least $2 \mathrm{~dB}$ as compared to the self-heterodyne transmission scheme. In the case of frequency selective channels, e.g. multi-path propagation, this improvement is shown to be much larger, because the presented approach is able to reconstruct the received subcarriers without the necessity of receiving all subcarriers.
\end{abstract}

\section{Introduction}

For the fifth generation mobile communications standard and also for future wireless local area network (WLAN) standards such as WiGig, mm-wave frequencies are considered and even frequencies in the terahertz regime might be of interest. Receive and transmit circuits at these frequencies are challenging to design and fabricate, especially for the active components. As an alternative to conventional superheterodyne receivers, self-mixing receivers, which employ a square-law detector for down-conversion from radio frequency (RF) into the baseband, are investigated in particular due to their low complexity. The main differences between these two receiver topologies are shown in Fig. 1. The superheterodyne approach requires a low noise amplifier (LNA), three mixers, one of which operates at RF and two at the intermediate frequency (IF), two local oscillators (LOs) for RF and IF, two analog-to-digital converters (ADCs) for the inphase and quadrature (IQ) signals and several filters. In comparison, the self-mixing receiver comprises only an LNA, one mixer, the square-law detector with only one input signal, an ADC and also some filters.

Apart from these obvious savings in the receiver circuitry, self-mixing receivers behave advantageously in several respects. First, they show a beneficial behavior when employed in multi-antenna systems. The receive signals from different antenna array elements are always superimposed in phase. As a result, receiver sensitivity is improved in fading channels with multi-path propagation (Shoji and Ogawa, 2004). Furthermore, self-mixing arrays increase the beamwidth compared to arrays which have an LO for downconversion, and thus make beam-forming superfluous in the 
(a)
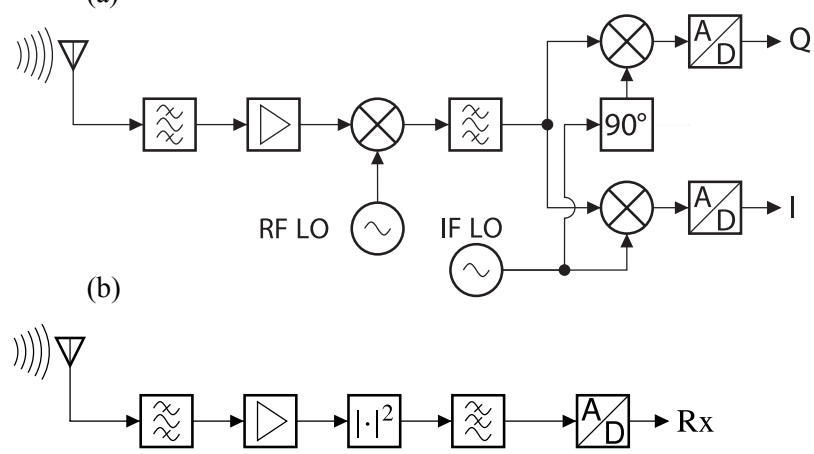

(c)

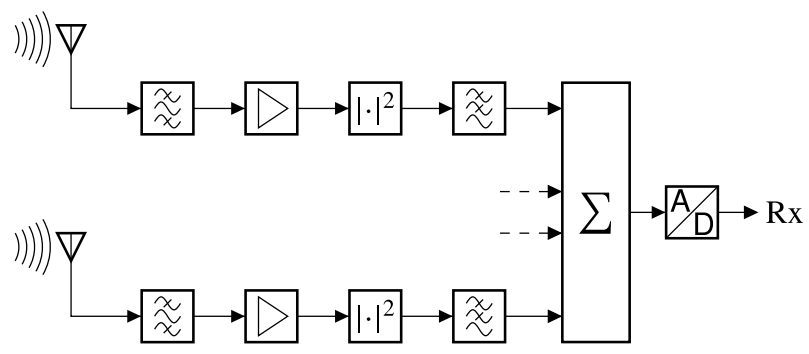

Figure 1. Comparison of different receiver topologies. (a) Superheterodyne receiver with IQ demodulation. (b) Self-mixing receiver with incoherent demodulation. (c) Self-mixing array with one A/D conversion.

receive case (Wang et al., 2015). So, the self-mixing receiver saves the hardware for multiple receive paths and following digital signal processing, as depicted in Fig. 1c. Second, the self-mixing receiver removes the need for an $\mathrm{LO}$ in the RF band. Hence, carrier frequency correction and carrier phase recovery are not necessary, which is very convenient in the mm-wave and terahertz regimes (Shoji et al., 2001; Foulon et al., 2014). Since all spectral components in the received RF signal are synchronous to, or in phase with, all other spectral components within the Rx band, the down-conversion is not influenced by any non-ideal behavior like phase noise of the RF LO. This is especially important for orthogonal frequency-division multiplexing (OFDM) signals, since the LO phase noise has a strong negative influence on the quality of recevied OFDM signals (Armada and Calvo, 1998; Pollet et al., 1995).

However, it is clear that incoherent demodulation introduces critical signal distortions as compared to conventional IQ-demodulation. This topic is also active under research for single carrier acoustic underwater communication systems (Wiedmann and Weber, 2013) and OFDM optical communication systems (Tan et al., 2013), where similar problems as for the self-mixing receivers arise. For analog single carrier amplitde modulation (AM) signals, it is known that a carrier has to be sent within the transmit signal having at least half of the transmit power for incoherent demodulation to yield the original desired signal (Kammeyer, 2011). The
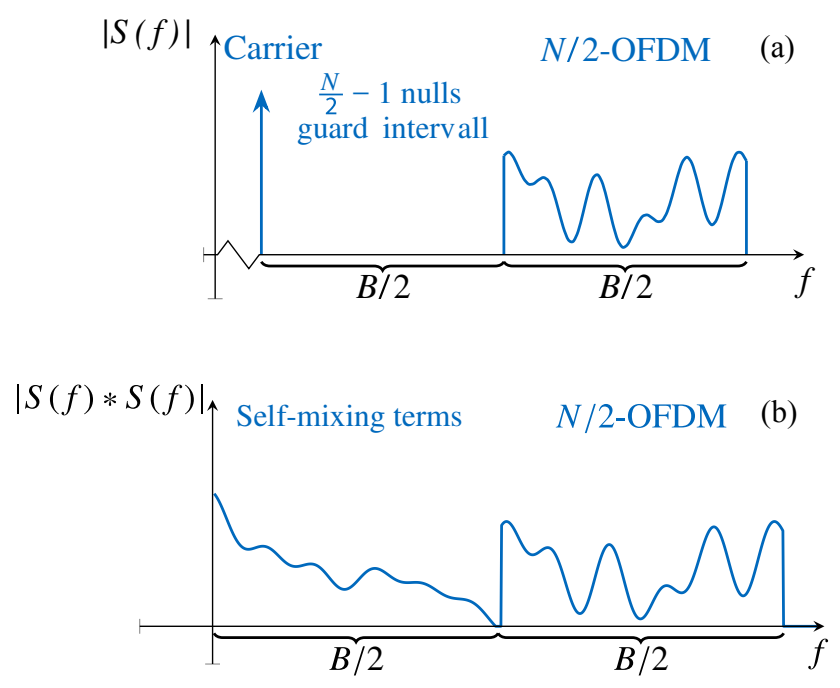

Figure 2. Down-conversion by selfmixing, as the working principle of the self-heterodyne transmission technique. (a) Self-heterodyne transmit signal. (b) Self-mixed self-heterodyne signal, containing the undistorted OFDM signal.

very same concept has been transfered to OFDM signals and is then called self-heterodyne (Shoji et al., 2001). The carrier and the OFDM signal are thereby spaced by a guard interval of the OFDM bandwidth $B / 2$, depicted in Fig. 2 for the magnitude transmit spectrum $|S(f)|$, such that the self-mixing products of the OFDM signal with the carrier are spectrally separated from the self-mixing products of the OFDM signal with itself. This is shown for the self-convoluted spectrum $|S(f) * S(f)|$. With the overall spectrum described as one OFDM symbol, it can be modeled by $N$-OFDM containing zeros in half of the bandwidth. Finally, the self-mixed signal just consists of an OFDM signal at an IF and can be further processed with standard IQ demodulation.

However, the self-heterodyne transmission scheme holds several disadvantages. The bandwidth efficiency and the power efficiency are only $50 \%$, since optimum performance is obtained with equal power distribution between the carrier and the OFDM subcarriers (Fernando et al., 2013b). This results in a difference in performance of about $5 \mathrm{~dB}$ between quadrature amplitude modulated (QAM) self-heterodyneOFDM and super-heterodyne QAM-OFDM, when the bit error ratio (BER) is considered over the signal to noise ratio (SNR) (Pacheco and Hatzinakos, 2004). A quite recent approach to avoid at least the poor bandwidth efficiency is the self-coherent receiver concept (Jin and Hong, 2016). This concept yet requires a more complicated analog RF circuitry.

Another important drawback is the strong dependence of the self-heterodyne concept on the transmitted carrier. This becomes crucial in frequency selective channels, e.g. with fading and multi-path propagation. Some solutions have been proposed, namely smart carrier positioning and subcarrier grouping and pairing (Fernando et al., 2013a, b; Choi et al., 
2011). Albeit, the problem persists that the receive signal strength depends on exactly one frequency, which is problematic if the carrier experiences deep fading.

In this paper, a novel technique for self-mixing signal recovery is presented that is capable of handling more general transmit signals. The necessity of a strong carrier signal for the down-conversion is dropped in favor of more bandwidth efficiency. In turn, the computational complexity of the signal reconstruction is increased. In this respect, this paper only aims at demonstrating a possibility for signal reconstruction, and not a solution ready for implementation in real time wireless applications.

In Sect. 2, we describe the general restrictions for the signal reconstruction of the transmit signal when utilizing a square-law detector for down-conversion. Based on this, two new transmission and reconstruction techniques are proposed in Sect. 3. Simulation results are discussed in Sect. 4 to demonstrate the superior behavior of the presented method over the self-heterodyne concept.

\section{Signal processing model of the self-mixing receiver}

The down-conversion of the received ( $\mathrm{Rx})$ signal is performed by a square law detector in time domain. This downconversion process will now be analyzed in detail, with a focus on OFDM-like transmit signals. This is not a really hard restriction, as any sampled and digitized signal is represented uniquely by a suitably sampled spectrum, which can be interpreted as an OFDM-like signal. More specific techniques such as pilot signals or specific implementation issues will not be discussed.

\subsection{Receiver description}

For the Rx signal analysis, a time domain transmit (Tx) signal is considered, which is represented in a sampled version as the vector $\boldsymbol{x}$, e.g. for one symbol. This signal passes through a channel, which is characterized by the sampled impulse response $\boldsymbol{h}$. After the channel, i.e. at the Rx antenna, white Gaussian noise $\boldsymbol{n}$ is added to model the thermal noise, leading to the received bandpass signal

$y=x * h+n$,

where the operator $*$ indicates temporal convolution. The low-pass signal which is measured and further processed is represented by the vector

$z=|\boldsymbol{y}|^{2}$,

where the absolute value operator $|\cdot|$ shall operate elementwise. This receiving procedure is depicted in Fig. 3a. The described noise model is only valid, if the gain of the LNA together with its noise figure is larger than the conversion loss of the mixer minus its noise figure. Otherwise, additive white noise has to be considered after the down-conversion,

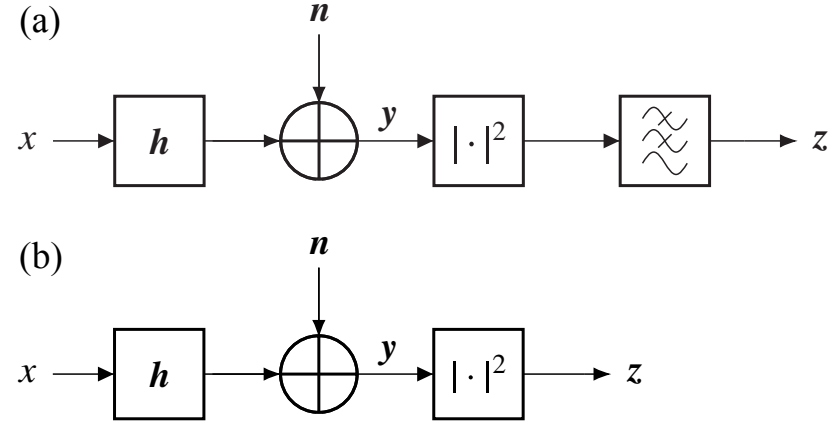

Figure 3. Signal processing models for the self-mixing receiver including channel and noise influence. (a) Self-mixing receiver model. (b) Self-mixing receiver model in the complex baseband.

too. The main difference between these noise models lies in the choice of the optimal constellation diagrams. With the presented noise model, standard QAM, phase-shift keying (PSK) or amplitude-shift keying (ASK) can be applied. With additive noise modelled after the square-law detector, a predistortion of constellation points in the IQ plane gives optimal results, as discussed by Wiedmann and Weber (2013).

The representation of this model in frequency domain will be analyzed in the following. The transmit signal is described by the frequency domain vector $\boldsymbol{X}=\mathcal{F}\{\boldsymbol{x}\}$, which is obtained via the discrete Fourier transform (DFT). The same holds for the channel matrix $\mathbf{H}=\mathcal{F}\{\boldsymbol{h}\}$, which is assumed to be diagonal due to a suitably chosen cyclic prefix. Therefore, the Rx signal before down-conversion is obtained as

$\boldsymbol{Y}=\mathbf{H} \boldsymbol{X}+\boldsymbol{N}=\mathcal{F}\{\boldsymbol{y}\}$

The Fourier transform of the multiplication is a selfconvolution, which needs to take into account the full spectrum up to the double frequency plus the signal bandwidth. This leads to the Rx spectrum

$\boldsymbol{Z}=\boldsymbol{Y} * \boldsymbol{Y}$

Therefore, low-pass filtering after the self-convolution is necessary to block the up-converted parts of the self-mixed signal. The self-mixed spectrum, as shown for a special case in Fig. $2 \mathrm{~b}$ in the lower half of the bandwidth $B$, is nearly triangular shaped if the Rx power is approximately evenly distributed over the signal bandwidth. As already observed in Fig. 2b, the spectrum is altered a lot in both amplitude and phase. For the down-converted part around DC, the convolution occurs between the terms at positive frequencies and the terms at negative frequencies, which show the same magnitude but are complex conjugated because the overall signal must be real valued in time domain.

This insight leads us to a much more convenient representation of the self-mixing receiving mechanism. The described convolution can be computed much easier, if just the complex baseband signal is considered, because low-pass filtering is 
not necessary, as depicted in Fig. 3b. This reduces the computational effort for simulations and also for reconstruction calculations. For all the following calculations, only complex baseband variables will be utilized before the square-law detector. The baseband Rx spectrum is then given as

$Z_{\ell}=\frac{1}{N} \sum_{k=0}^{N-1} Y_{k} Y_{k+\ell}^{*}, \quad \ell=-N+1, \ldots, N-1$,

dependent on the spectral components $Y_{k}$ of the complex baseband signal, represented by the vector $\boldsymbol{Y}$ in frequency domain with $N$ non-zero entries from $k=0$ to $k=N-1$. The star operator $(\cdot)^{*}$ denotes the complex conjugate. For the output signal, the index $\ell$ defines the baseband frequency as $f_{\ell}=\ell / T_{\mathrm{S}}$, where $T_{\mathrm{S}}$ is the symbol duration, for positive and negative values of $\ell$.

\subsection{Consequences for the signal recovery}

One consequence of the self-mixing is that the resulting spectrum occupies twice as much bandwidth as compared to the complex baseband signal. Equation (5) shows the corresponding property in frequency domain, i.e. that the spectrum shows a complex conjugated symmetry. Finally, the sum in Eq. (5) itself can be identified as an autocorrelationalike function, in frequency domain as opposed to the more common formulation in time domain. It follows that the selfmixed time domain signal is correctly described in equivalence to the power spectral density

$\boldsymbol{z}=\left[\begin{array}{lllll}y_{1} y_{1}^{*} & y_{2} y_{2}^{*} & \ldots & y_{2 N-2} y_{2 N-2}^{*} & y_{2 N-1} y_{2 N-1}^{*}\end{array}\right]^{\mathrm{T}}$,

with a suitably chosen oversampling. Hence, Eq. (6) represents a more precise version of Eq. (2). This gives us the insight that an oversampling by a factor of two is necessary in the down-converted signal to be able to extract the full available spectral information. If the oversampling criterion is not met, the absolute values of the time domain signal $\boldsymbol{y}$ are still obtained as described by Eq. (2). However, not all possible information is acquired, as the self-mixed signal contains more information than just the amplitude in time domain. Tracing the presented problem in Eq. (6) back to the phase-retrieval problem, even though it is typically formulated for phase-less amplitude only measurements in frequency domain or in optical Fourier domains, leads to the insight that the phase can not take arbitrary values because the original spectrum $\boldsymbol{Y}$ has compact support, i.e. its bandwidth is limited to $B$ sampled at $N$ frequency points. This leads us to the conclusion that $2^{N}$ valid solutions exist with $N$ sampling points for one symbol (Klibanov et al., 1995).

Therefore, Eq. (5) is interpreted as complex valued system of polynomial equations containing the additional information. Due to its symmetry, it provides $N$ independent complex equations, e.g. for positive frequencies with $\ell \geq 0$ only. By splitting real and imaginary part of the measured vector $\boldsymbol{Z}, 2 N$ real valued equations can be obtained, in addition to the $2 N-1$ real valued time domain equations. This equation system in frequency is not directly solvable with a large number of unknowns, and also might not lead to a unique solution. Hence, an iterative solution procedure has to be applied to reconstruct the transmit signal for general self-mixed Rx signals from the presented equations. A suitable formulation might be to aim for the reconstruction of the frequency domain vector $\boldsymbol{Y}$, because it can be employed as an unknown vector for both time and frequency domain $\mathrm{Rx}$ signals. The frequency domain equation systems are already contained in Eq. (6), for $\ell \geq 0$ only. The time domain signal comprising the amplitude-only measurement data is attained as

$\boldsymbol{z}=|\boldsymbol{y}|^{2}=|\mathbf{W} \boldsymbol{Y}|^{2}$,

by the application of the DFT matrix $\mathbf{W}$ and a following element-wise absolute-value-squaring-operation. From a theoretical point of view, it was discussed earlier that the time domain signal should be oversampled to correctly model the self-mixed spectrum. Due to the bandwidth-limitation of the vector $\boldsymbol{Y}$, oversampling in $\boldsymbol{z}$ is not strictly necessary anymore in Eq. (7). An oversampled representation of $z$ could even be counterproductive since it requires a resampling of the unknown vector $\boldsymbol{Y}$ or $\mathbf{W} \boldsymbol{Y}$ in each solver iteration, if an iterative solution strategy is applied for the signal reconstruction.

Signal analysis shows that the self-mixed Rx signal contains further degrees of freedom (DOFs) even if optimally measured (with a suitable oversampling) and the underlying equation systems do not offer unique solutions. These remaining DOFs can thus be represented by $N$ phases in time domain, where it has to be kept in mind that only $2^{N}$ valid solutions exist. The not uniquely solvable polynomial equation system in frequency domain is also an implication of the extra DOFs. Restrictions or a-priori knowledge concerning the Tx signal can help to pose the presented signal reconstruction problem better and even to offer unique solutions, which is of course desirable for information transmission.

\section{Signal reconstruction techniques with amplitude-shift-keying modulation}

The remaining task is to reconstruct the original receive signal $\boldsymbol{y}$ from the knowledge of its magnitude only, represented by sufficiently oversampled measurements of $z$. To circumvent the ambiguity of the recovered signal, the main concept is that information about the transmit signal is known a-priori to eliminate the extra DOFs for the reconstruction of the signal. Two different approaches, one based on the time domain signal representation and one based on the frequency domain representation, will be discussed in the following. 


\subsection{Time-domain multiplexing}

As a quite intuitive approach, the transmitted information is coded in the magnitude of the time domain signal. At each sampling point in time, the transmitted information is encoded in the envelope of the time domain signal with ASK modulation. This requires no reconstruction effort, as the data for each timestep is directly read from the receveid time domain signal. The extra DOFs in the Rx signal, i.e. the phases of the time domain signal, are simply ignored. As the separation of the transmitted information is achieved over time, this approach is called time-domain multiplexing (TDM).

Nevertheless, this approch works only well if the transmission channel, e.g. represented by a diagonal matrix $\mathbf{H}$, has about the same influence on all frequencies within the transmit bandwidth. If this is not the case, predistortion or equalization according to a channel estimation have to be considered. In the scope of this paper, such methods have not been considered.

\subsection{Frequency-domain multiplexing}

A similar concept is investigated in frequency domain. An OFDM signal with ASK for all the subcarriers is considered. Thereby, the phases of all OFDM subcarriers $\varphi=\measuredangle \boldsymbol{Y}$ are defined in advance by assigning a random phase value to each subcarrier and just the magnitudes of the subcarriers are employed for information transmission. As compared to the common QAM modulation, the ASK modulation is only performed on the positive part of the I-axis. Therefore, the distance between valid points in the constellation diagrams is smaller for ASK modulation. However, since only half of the transmit power is put into the data symbols for the selfheterodyne concept, the ASK modulation can still perform advantegeously.

For the reconstruction of the original transmit signal, different solution strategies can be applied, as discussed in Sect. 2. For the following simulations, a solution is obtained with the help of the algorithm of Gerchberg and Saxton (1972). This algorithm iteratively fulfills both the time domain and the frequency domain constraints. In one iteration, the measured time domain signal together with the time domain phase vector $\measuredangle \mathcal{F}\{\boldsymbol{Y}\}$ obtained by Fourier transform of the spectrum forms the reconstructed time domain signal signal. In the second step of this iteration, Fourier transform is applied to the time domain signal. In the resulting spectrum, the phase values are replaced by the a-priori information about the phases $\varphi$.

For the case of a frequency-dependent channel, the channel properties are assumed to be known at the receiver. Therefore, the influence of the channel on the receive signal can be corrected during the frequency-domain phase of the Gerchberg-Saxton algorithm. In each iteration, the phase values are corrected by the phases of the spectrum of the channel $\measuredangle \boldsymbol{h}$. The processed spectrum is corrected by the amplitudes $|\boldsymbol{h}|$.

\section{Simulation results}

To demonstrate the working principle of the proposed concepts, Monte Carlo simulations have been carried out. Thereby, the BER was analyzed for different SNRs. Thereby, the receiver model depicted in Fig. 3 with a channel impulse response, a square-law detector in time domain and low-pass filtering has been employed. An OFDM signal with 1024 subcarriers is considered, which defines the available bandwidth. Per OFDM symbol, 4096 bit are represented, i.e. 4 bit per subcarrier for standard OFDM. For modulation, 16ASK is utilized for standard OFDM (ASK-OFDM) and selfmixing OFDM (OFDM-SM). The time domain signal is also represented by 1024 samples. Hence, also the TDM concept needs to have 4 bit per sample to achieve the same data throughput, i.e. also 16-ASK modulation per sample for the TDM self-mixing (TDM-SM) approach. In comparison, the self-heterodyne transmission scheme is analyzed. Since only 512 subcarriers are utilized for the payload, 256-QAM has been chosen for 8 bit per subcarrier to have the same data rate for the self-heterodyne concept (Self-Het.) as for the other simulated cases. Moreover, a 256-QAM-OFDM signal with only half the bandwidth, i.e. 512 subcarriers, is simulated as a reference. For comparison, the constellation diagrams, for symbols with the same average transmit power, are given in Fig. 4a. The distance between nearest neighbours in the constellation diagrams is about $68 \%$ for ASK as compared to QAM.

For the sake of clarity, also the constellation diagrams for a lower modulation order, 8-ASK and 64-QAM, are depicted in Fig. 4b. In this case, the distance between nearest neighbouring symbols in the constellation diagrams is about $54 \%$ for ASK as compared to QAM. These examples already show the advantage of ASK modulation over QAM modulation schemes for the self-mixing receiver, because the selfheterodyne concept only uses half the transmit power for the data symbols. Therefore, the ASK constellation points exhibit a larger distance which should lead to a better BER at the same SNR level.

For the two different modulation orders, the BER has been computed for different receive SNR levels for an AWGN channel, without any further influence on the received spectrum. This is shown in Figs. 5 and in 6 for the lower order modulated signals. The known difference in performance of $5 \mathrm{~dB}$ between 256-QAM-OFDM and Self-Het. is observed (Pacheco and Hatzinakos, 2004). The common superheterodyne ASK-OFDM approach performs about $2 \mathrm{~dB}$ better than the Self-Het. approach, which confirms the previous reflections. The TDM-SM and OFDM-SM schemes almost achieve the performance of standard ASK-OFDM with a super-heterodyne receiver. The remaining small difference 


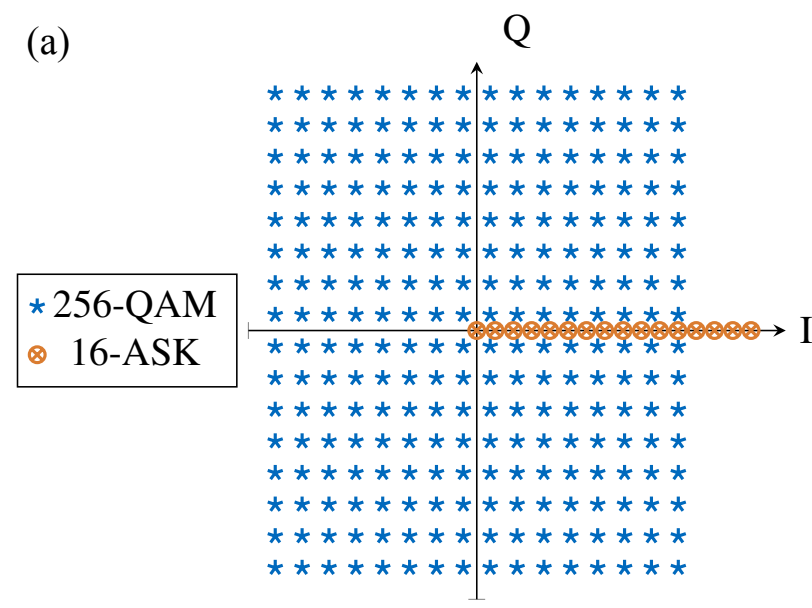

(b)

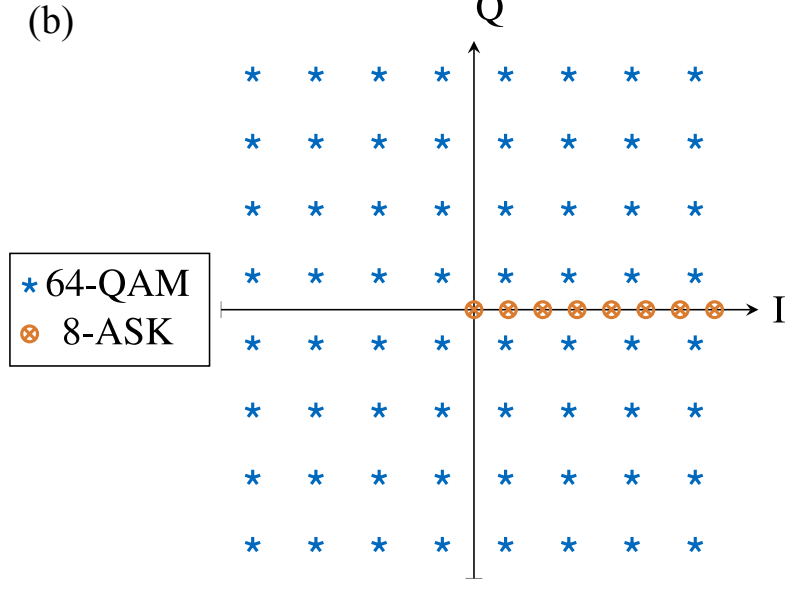

Figure 4. Constellation diagram comparison for the normal OFDM versions with QAM and ASK modulation, self-heterodyne OFDM with QAM and self-mixing with ASK modulation for the same average transmit power per symbol. (a) Comparison of 256-QAM and 16-ASK. (b) Comparison of 64-QAM and 8-ASK.

is probably due to the different noise behavior, and, in case of OFDM-SM, the computational errors when solving the selfmixing equation system.

A further example will demonstrate the advantages of the proposed method. Now, a constant AWGN channel is considered, where, however, the frequency of the transmitted carrier of the self-heterodyne scheme is damped by a factor of 0.1 . The impulse and frequency response of the channel are assumed to be known at the receiver. Thus, they are corrected without the need of considering pilot signals. Monte Carlo simulation results are presented in Fig. 7. This leads to more than $10 \mathrm{~dB}$ difference in performance at a BER of $10^{-2}$ between ASK-OFDM and self-heterodyne. Again, the OFDM-SM approach performs almost as well as the common ASK-OFDM. Only the TDM-SM approach is worse than self-heterodyne for large SNR, because the time domain signal is strongly distorted by the frequency selective chan-

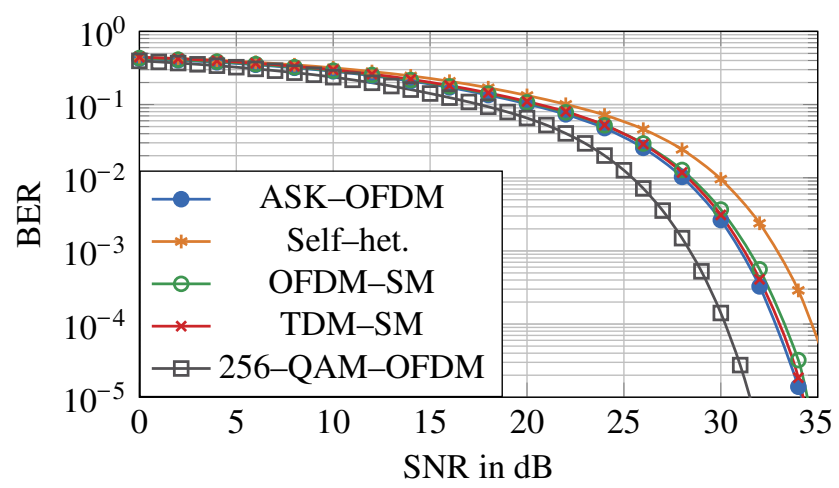

Figure 5. BER simulation results for the comparison of standard ASK-OFDM, the self-heterodyne transmission scheme and the two proposed transmission schemes with AM and multiplexing in time and frequency domain.

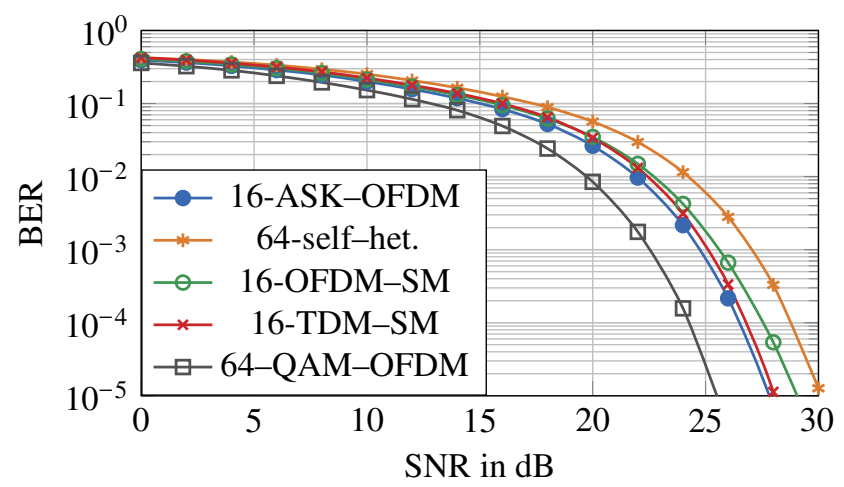

Figure 6. BER simulation results for the comparison of standard ASK-OFDM, the self-heterodyne transmission scheme and the two proposed transmission schemes with AM and multiplexing in time and frequency domain, for the lower order modulation schemes.

nel. This can be avoided by equalization/predistortion, which is not considered here. For ASK-OFDM and OFDM-SM, it can be seen that only one of 1024 subcarriers is not useful for the signal transmission, as a saturation at high SNR is observed. As opposed to this, the self-heterodyne transmission does not work in a satisfying manner anymore with only one subcarrier experiencing an attenuation.

As a final example, a two-path channel is considered. This channel exhibits a direct line of sight connection with an amplitude of 1 and a slightly attenuated reflected component with an amplitude of 0.8 and with a longer runtime. One minimum of this fading transmission channel lies at the self-heterodyne carrier frequency. The BER simulation results are presented in Fig. 8. The self-heterodyne approach shows about $9 \mathrm{~dB}$ worse performance than ASK-OFDM. The TDM-SM method without channel predistortion or equalization only gives random results at a BER of about 0.5. The self-mixing signal recovery for OFDM-SM is shown to yield excellent results, as the original signal can almost perfectly be reconstructed. 


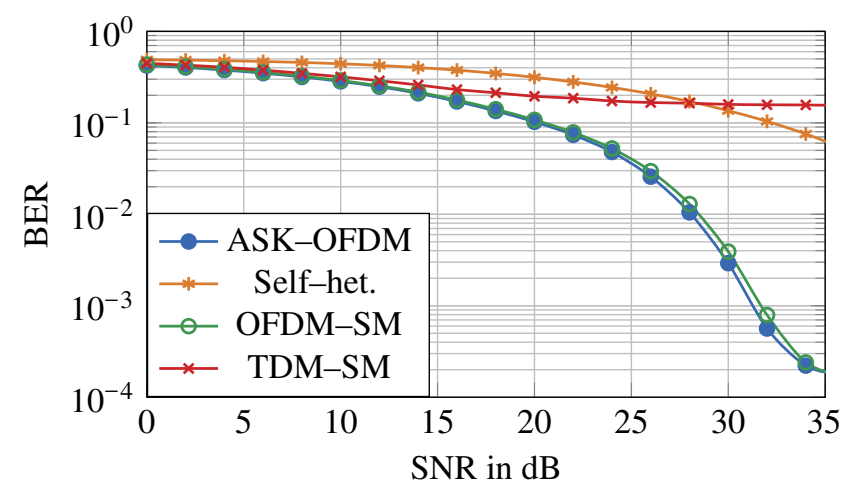

Figure 7. BER simulation results with a frequency selective channel, damping the self-heterodyne carrier frequency by a factor of 10 .

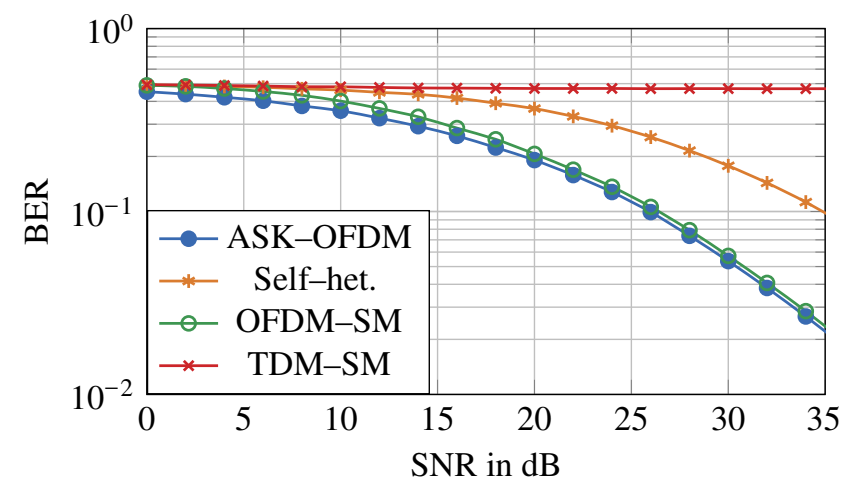

Figure 8. BER simulation results for a two-path fading channel, with a direct LOS connection with amplitude 1 and a reflected component with amplitude 0.8 .

\section{Conclusions}

A complex baseband description of the self-mixing receiver, or square law detector, has been proposed, which was shown to be very useful for the signal recovery. Two non-linear equation systems condensing the available information for the signal reconstruction have been obtained. Additionally, the general limitations of the self-mixing concept, as compared to a common super-heterodyne receiver, have been analyzed with these equation systems. It was found that parts of the transmit signal have to been known at the receiver for the possibility of a unique signal reconstruction after self-mixing and information transmission.

With the proposed method, the standard IQ demodulation as for super-heterodyne and self-heterodyne receivers has not been employed, but the signal has been reconstructed with the help of the presented equation systems. Thereby, the power-efficiency was increased as compared to the selfheterodyne transmission scheme and the strong dependence on one spectral component was eliminated, as verified in Monte Carlo simulations. Hence, the BER performance of the presented approach was shown to be much better than for the self-heterodyne transmission scheme, in particular for fading and frequency-selective channels. This comes at the cost of increased computational effort, while maintaining the known advantages of self-mixing receivers.

Data availability. The underlying research data can be requested from the authors.

Competing interests. The authors declare that they have no conflict of interest.

Acknowledgements. This work was supported by the German Research Foundation (DFG) under grant EI 352/17-1.

This work was supported by the German Research Foundation (DFG) and the Technische Universität München within the funding programme Open Access Publishing.

Edited by: Jens Anders

Reviewed by: Emanuele Viterbo and one anonymous referee

\section{References}

Armada, A. G. and Calvo, M.: Phase noise and sub-carrier spacing effects on the performance of an OFDM communication system, IEEE Commun. Lett., 2, 11-13, 1998.

Choi, C.-S., Shoji, Y., and Ogawa, H.: Implementation of an OFDM baseband with adaptive modulations to grouped subcarriers for millimeter-wave wireless indoor networks, IEEE Trans. Consum. Electron., 57, 1541-1549, 2011.

Fernando, N., Hong, Y., and Viterbo, E.: Self-heterodyne OFDM transmission for frequency selective channels, IEEE Trans. Commun., 61, 1936-1946, 2013a.

Fernando, N., Hong, Y., and Viterbo, E.: Subcarrier pairing for selfheterodyne OFDM, in: IEEE Int. Conf. Commun. (ICC), $2013 \mathrm{~b}$.

Foulon, S., Pruvost, S., Pache, D., Loyez, C., and Rolland, N.: A $140 \mathrm{GHz}$ multi-gigabits self-heterodyne transceiver for chip-tochip communications, in: 44th Eur. Microwave Conf., 2014.

Gerchberg, R. W. and Saxton, W. O.: A practical algorithm for the determination of phase from image and diffraction plane pictures, Optik, 35, 237-246, 1972.

Jin, Q. and Hong, Y.: Self-coherent OFDM with undersampling downconversion for wireless communications, IEEE Trans. Wireless Commun., 15, 6979-6991, 2016.

Kammeyer, K.-D.: Nachrichtenübertragung, Vieweg+Teubner Verlag, Wiesbaden, 5 edn., 2011.

Klibanov, M. V., Sacks, P. E., and Tikhonravov, A. V.: The phase retrieval problem, Inverse Problems, 11, 1-28, 1995.

Pacheco, R. A. and Hatzinakos, D.: BER analysis of self-heterodyne OFDM transmission scheme, in: Can. Conf. Elect. and Comput. Eng. (IEEE), 4, 1953-1956, 2004. 
Pollet, T., Van Bladel, M., and Moeneclaey, M.: BER sensitivity of OFDM systems to carrier frequency offset and Wiener phase noise, IEEE Trans. Commun., 43, 191-193, 1995.

Shoji, Y. and Ogawa, H.: 70-GHz-band MMIC transceiver with integrated antenna diversity system: application of receivermodule-arrayed self-heterodyne technique, IEEE Trans. Microwave Theory Tech., 52, 2541-2549, 2004.

Shoji, Y., Nagatsuka, M., Hamaguchi, K., and Ogawa, H.: 60 GHz band 64 QAM/OFDM terrestrial digital broadcasting signal transmission by using millimeter-wave self-heterodyne system, IEEE Trans. Broadcast., 47, 218-227, 2001.
Tan, Z., Shao, Y., Sun, Y., Wang, S., and Luo, Y.: 64QAM-OFDMbased millimeter-wave access system using DMT modulation and electrical self-mixing, Microwave Opt. Technol. Lett., 56, 259-262, 2013.

Wang, K., Wächter, T. J., Hartmuth, H., Fei, H., Hamberger, G. F., and Eibert, T. F.: Self-mixing antenna arrays with wide receiving angular range, in: IEEE Int. Symp. Antennas and Propagation \& USNC/URSI Nat. Radio Sci. Meeting, 647-648, 2015.

Wiedmann, K. and Weber, T.: Constellation-constrained capacities for parametric underwater communication, in: OCEANS Bergen, MTS/IEEE, 1-7, 2013. 